\title{
Commentary
}

\section{Metabolic Resuscitation Therapy for Sepsis: Where to Go}

\author{
Zhanguo Liu, Ping Chang* \\ Department of Critical Care Medicine, Zhujiang Hospital, Southern Medical University, Guangzhou, China
}

\author{
ARTICLE INFO \\ Article History \\ Received 29 March 2021 \\ Accepted 09 June 2021 \\ Keywords \\ Sepsis \\ metabolic resuscitation \\ vitamin C \\ hydrocortisone \\ thiamine
}

(c) 2021 First Affiliated Hospital of Zhengzhou University. Publishing services by Atlantis Press International B.V. This is an open access article distributed under the CC BY-NC 4.0 license (http://creativecommons.org/licenses/by-nc/4.0/).
Sepsis is a life-threatening condition with a high incidence, mortality, and cost. It is recognized as a primary priority by the World Health Organization [1]. There is an urgent need for a safe, effective, and inexpensive treatment. A retrospective clinical study has recently found that hydrocortisone combined with ascorbic acid (vitamin C) and thiamine (HAT therapy), also called as metabolic resuscitation therapy, can significantly reduce mortality and improve organ dysfunction in patients with sepsis [2]. Marik showed that sepsis management should include early diagnosis and timely treatment with antibiotics, source control, and a conservative approach, including fluid management, the administration of vasopressors, and early use of metabolic resuscitation drugs, including vitamin $\mathrm{C}$, hydrocortisone, and thiamine [3]. This notion has caused widespread concern. Theoretically, these drugs may improve sepsis by inhibiting an excessive inflammatory response, reversing shock, restoring organ perfusion, and promoting cell metabolism recovery [4]. Some Randomized Controlled Trials (RCTs) have been conducted to validate the efficacy of metabolic resuscitation drugs alone or in combination [4].

Unlike previous sepsis studies, the primary outcome in most RCTs using metabolic resuscitation drugs for treating sepsis has been transformed from mortality to improvement in the Sequential Organ Failure Assessment (SOFA) score or the shortening of septic shock duration. The cause of mortality in patients with sepsis is complicated; undoubtedly, these transformations can reduce the confusion caused by some competing mortality risks and directly reflect the improvement of organ function. However, in most of published RCTs, except the ORANGES study, which demonstrated a shorter duration of vasopressor use in patients

"Corresponding author. Email: changp963@163.com

Peer review under responsibility of the First Affiliated Hospital of Zhengzhou University with HAT therapy [5], the SOFA score or shock status of patients with sepsis with metabolic resuscitation drugs alone or in combination has not showed any excepted improvement. Two small-sample RCTs showed that vitamin C could positively impact organ function and reverse shock [6,7]. Some intensivists believe that RCTs do not replicate real-world efficacy. This belief may be related to different exclusion criteria, including patient heterogeneity, non-standardized joint intervention, and different dose ranges and timings of the three drugs [8]. It is also imperfect to choose SOFA score improvement or the reversal of septic shock as the primary outcome of the efficacy of metabolic resuscitation drugs. The endpoint SOFA score may not be available for patients who died or were ICU-discharged earlier. This situation can lead to attrition bias and underestimating the true benefit of metabolic resuscitation drugs [9]. Regarding the reversal of shock as the primary outcome, mortality during the study period may also affect the duration of vasopressors. The reasons mentioned above probably affect the trial results.

A series of negative outcomes in RCTs have not made intensivists lose heart and enthusiasm for vitamin C, hydrocortisone, and thiamine [8]. Evaluations of the effectiveness of these drugs alone or in combination in patients with sepsis will certainly continue for a long time. To better detect the benefits of vitamin C, hydrocortisone, and thiamine in patients with sepsis and avoid wasting trial resources, it is time to summarize and make changes. First, the current negative outcomes cannot reflect the economic effect of the three drugs. In addition, the low cost and continued availability of vitamin $\mathrm{C}$, hydrocortisone, and thiamine are important reasons to stimulate widespread interest among intensivists [2]. Therefore, some pharmacoeconomic analysis maybe necessary, such as cost-effectiveness, to evaluate the cost and benefit of these drugs. Cost-effectiveness is an analytic tool in which a certain 
therapy and at least one alternative treatment are compared in terms of cost per unit of effectiveness [10]. Effects include health outcomes, such as Quality-adjusted Life-Years (QALY), rather than monetary measures, as in cost-analysis, proposed as an outcome measure for critical care trials [11]. By comparing the cost of generating one additional QALY, we can determine whether these resuscitation drugs are more cost effective and worthwhile than placebo. Second, the ORANGES study showed that HAT therapy can shorten vasopressor duration in patients with sepsis. It may also be related to its performance in two non-teaching hospitals because its minimal resource utilization better reflects real-world clinical management [5]. Therefore, recruiting some patients in non-teaching hospitals may help to reduce the interference of other combination treatments. Third, sepsis is an overly heterogeneous syndrome with different subsets or phenotypes, resulting in different risks for poor outcomes and different responses to some interventions, such as glucocorticoid and fluid resuscitation [12-14]. Based on this feature, sepsis should be managed by stratifying patients into subphenotypes, in which different types can be targeted depending on the intervention strategy. Accordingly, it is crucial for the future RCTs to identify the subclass of sepsis and its responses to the resuscitation drugs through a secondary analysis of published RCTs. Lastly, even if these drugs cannot improve sepsis prognosis, their efficacy in preventing patients with suspected infection in the ICU or emergency room from suffering sepsis or septic shock is still unclear, thus, an urgent need for its determination.

In conclusion, metabolic resuscitation therapy in patients with sepsis has not yet been established, and beneficial evidence of metabolic resuscitation drugs for sepsis is still urgently expected. However, maybe it is time to make adjustments to the protocol of future RCTs on metabolic resuscitation therapy.

\section{CONFLICTS OF INTEREST}

The authors declare they have no conflicts of interest.

\section{FUNDING}

This work is supported by the National Natural Science Foundation of China (Grant 81971859 and 81971883), the Natural Science Foundation of Guangdong Province, China (Grant 2019A1515011856).

\section{REFERENCES}

[1] Reinhart K, Daniels R, Kissoon N, Machado FR, Schachter RD, Finfer S. Recognizing sepsis as a global health priority-a WHO resolution. N Engl J Med 2017;377;414-17.

[2] Marik PE, Khangoora V, Rivera R, Hooper MH, Catravas J. Hydrocortisone, vitamin $\mathrm{C}$, and thiamine for the treatment of severe sepsis and septic shock: a retrospective before-after study. Chest 2017;151;1229-38.

[3] Marik PE. The management of sepsis: science \& fiction. J Thorac Dis 2020;12;S1-S4.

[4] Moskowitz A, Andersen LW, Huang DT, Berg KM, Grossestreuer AV, Marik PE, et al. Ascorbic acid, corticosteroids, and thiamine in sepsis: a review of the biologic rationale and the present state of clinical evaluation. Crit Care 2018;22;283.

[5] Iglesias J, Vassallo AV, Patel VV, Sullivan JB, Cavanaugh J, Elbaga Y. Outcomes of metabolic resuscitation using ascorbic acid, thiamine, and glucocorticoids in the early treatment of sepsis: the ORANGES trial. Chest 2020;158;164-73.

[6] Fowler AA, Syed AA, Knowlson S, Sculthorpe R, Farthing D, DeWilde C, et al. Phase I safety trial of intravenous ascorbic acid in patients with severe sepsis. J Transl Med 2014;12;32.

[7] Zabet MH, Mohammadi M, Ramezani M, Khalili H. Effect of high-dose ascorbic acid on vasopressor's requirement in septic shock. J Res Pharm Pract 2016;5;94-100.

[8] Marik PE, Varon J, Surani SR. Hydrocortisone, ascorbic acid and thiamine for sepsis: is the jury out?. World J Diabetes 2020;11;90-4.

[9] Marik PE, Payen D. CITRIS-ALI: how statistics were used to obfuscate the true findings. Anaesth Crit Care Pain Med 2019;38;575-7.

[10] Sanders GD, Neumann PJ, Basu A, Brock DW, Feeny D, Krahn M, et al. Recommendations for conduct, methodological practices, and reporting of cost-effectiveness analyses: second panel on costeffectiveness in health and medicine. JAMA 2016;316;1093-103.

[11] Barrett KA, Hawkins N, Fan E. Economic evaluation of venovenous extracorporeal membrane oxygenation for severe acute respiratory distress syndrome. Crit Care Med 2019;47;186-93.

[12] Seymour CW, Kennedy JN, Wang S, Chang CCH, Elliott CF, Xu Z, et al. Derivation, validation, and potential treatment implications of novel clinical phenotypes for sepsis. JAMA 2019;321;2003-17.

[13] Zhang Z, Zhang G, Goyal H, Mo L, Hong Y. Identification of subclasses of sepsis that showed different clinical outcomes and responses to amount of fluid resuscitation: a latent profile analysis. Crit Care 2018;22;347.

[14] Zhang Z, Pan Q, Ge H, Xing L, Hong Y, Chen P. Deep learningbased clustering robustly identified two classes of sepsis with both prognostic and predictive values. EBioMedicine 2020;62;103081. 